\title{
Cold extraction of phenolic compounds from watercress by high hydrostatic pressure: Process modelling and optimization
}

\author{
José Pinela $^{\mathrm{a}, \mathrm{b}}$, M.A. Prieto ${ }^{\mathrm{a}, \mathrm{c}}$, Lillian Barros $^{\mathrm{a}}$, Ana Maria Carvalho ${ }^{\mathrm{a}}$, M. Beatriz P.P. Oliveira ${ }^{\mathrm{b}}$, \\ Jorge A. Saraiva ${ }^{\mathrm{d}}$, Isabel C.F.R. Ferreira ${ }^{\mathrm{a}, *}$ \\ a Centro de Investigação de Montanha (CIMO), Instituto Politécnico de Bragança, Campus de Santa Apolónia, 5300-253 Bragança, Portugal \\ ${ }^{\mathrm{b}}$ REQUIMTE/LAQV, Faculdade de Farmácia, Universidade do Porto, Rua Jorge Viterbo Ferreira, $n^{\circ}$ 228, 4050-313 Porto, Portugal \\ ${ }^{\mathrm{c}}$ Nutrition and Bromatology Group, Faculty of Food Science and Technology, University of Vigo, Ourense Campus, E32004 Ourense, Spain \\ ' QOPNA, Departamento de Química, Universidade de Aveiro, Campus Universitário de Santiago, 3810-193 Aveiro, Portugal
}

\section{A R T I C L E I N F O}

\section{Keywords:}

Nasturtium officinale

Phenolic acids

Flavonoids

High hydrostatic pressure

Extraction optimization

\begin{abstract}
A B S T R A C T
High hydrostatic pressure (HHP) was applied to the extraction of phenolic compounds from watercress (Nasturtium officinale). The process was optimized by response surface methodology using a five-level central composite design combining the independent variables of processing time $(t, 1.5-33.5 \mathrm{~min})$, pressure ( $P$, 0.1-600 MPa) and solvent $(S, 0-100 \%$ of ethanol, v/v). The individual and grouped phenolic compounds, analyzed by HPLC-DAD-ESI/MS, and the extraction yield were used as response variables. The theoretical models were fitted to the experimental data, statistically validated, and used in the prediction and optimization steps. The optimal HHP conditions for the extraction of phenolic compounds were: $t=3.1 \mathrm{~min}, P=600 \mathrm{MPa}$ and $S=100 \%$, and originated $64.68 \pm 2.97 \mathrm{mg} / \mathrm{g}$ of extract. This study highlighted the HHP as a promising technology to cold extract phenolic compounds (phenolic acids and flavonoids) from watercress in a selective way using a green solvent and reduced extraction times.
\end{abstract}

\section{Introduction}

The recovery of bioactive compounds from natural sources and their further incorporation into foods [1], dietary supplements [2] and cosmeceuticals [3], either in isolated form or in enriched extracts, is a current hot topic that involves many research fields. Phenolic compounds are among the most desired plant secondary metabolites because of their recognized bioactivities and capacity to protect against free radical-mediated diseases [4]. Several studies have been carried out in recent years to improve the extraction of these compounds from plant materials [5-7], but more efficient and sustainable methods need to be developed to achieve higher yields and superior quality products at lower processing costs.

Watercress (Nasturtium officinale R. Br.) is a semi-aquatic fastgrowing plant of the Brassicaceae family with recognized health-promoting effects. Its consumption in a daily diet has been linked with a reduced risk of chronic diseases including different types of cancer [8-11]. This species is an interesting source of pharmacologically active phytochemicals [12-14] whose involvement in antigenotoxic and anticancer processes has been demonstrated in both in vivo and in vitro assays [11,15-17]. A previous study reported $p$-coumaric acid, quercetin-3-O-sophoroside and isorhamnetin-O-hydroxyferuloylhexoside- $O$ hexoside as the most abundant phenolic compound in wild watercress [14]. Higher concentrations of flavonoids than phenolic acids were reported in these extracts due to the high contents of isorhamnetin and quercetin glycosides and, in lesser extent, of kaempferol [14]. In turn, a dimer of caffeoylmalic acid, disinapoylgentibiose and ferulic acid were identified as the predominant polyphenols in watercress juice, which demonstrated capacity to inhibit digestive enzymes relevant to type 2 diabetes and obesity [12]. Despite the great potential of these compounds in various industrial sectors, the development of more efficient processes for their recovery from watercress and other natural sources remains challenging.

High hydrostatic pressure (HHP) is an emerging technology increasingly used in the food industry as a cold pasteurization method [18-21]. It consists on subjecting packaged or in bulk foods to pressures up to $1000 \mathrm{MPa}$ inside a vessel filled with water, fluid that acts as pressure-transmitting medium $[18,21]$. During processing, the pressure is transmitted in an isostatic and quasi-instantaneous manner throughout the sample, which makes the processing time independent

\footnotetext{
Abbreviations: Igd, isorhamnetin glycoside derivatives; $K g d$, kaempferol glycoside derivatives; $P$, pressure; $Q g d$, quercetin glycoside derivatives; $S$, solvent; $T$, processing time

* Corresponding author.

E-mail address: iferreira@ipb.pt (I.C.F.R. Ferreira).
} 


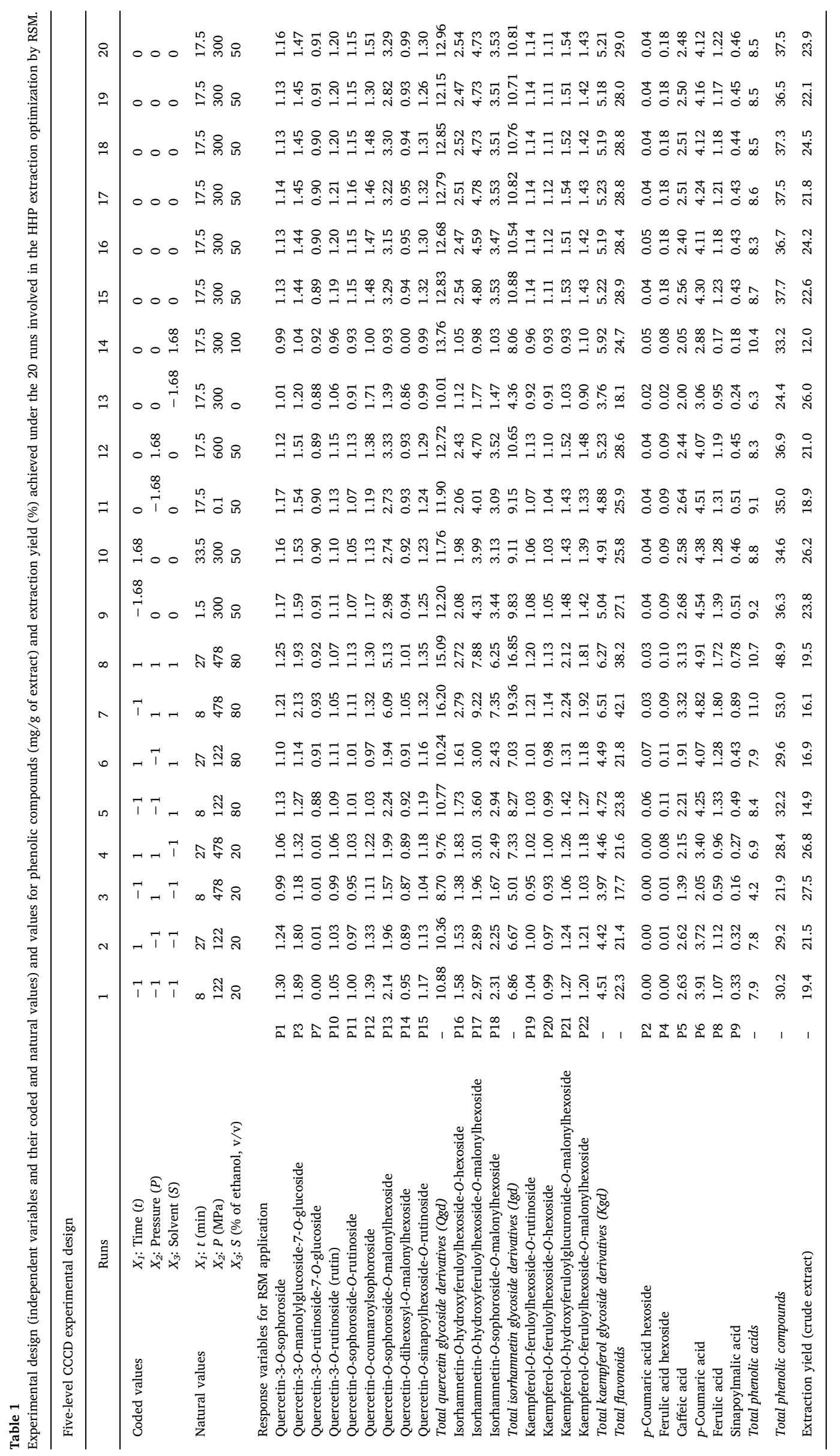


of the sample shape or size. In addition, the temperature increase with increasing pressure is minimal $\left(-3{ }^{\circ} \mathrm{C} / 100 \mathrm{MPa}\right)[22,23]$, thus being a good alternative to heat-based treatments.

HHP has been explored for some time by the food industry. Its application for extraction of high added-value compounds from plant materials is relatively recent and very promising, but more research focusing on different compounds and plant materials is still needed. The applied pressure promotes the rupture of the plant tissues, cell walls and organelles, a phenomenon that enhances the mass transfer of the solvent into the sample and of compounds to the solvent [24]. In addition, the higher the hydrostatic pressure is, the more solvent can enter cells and the more compounds can permeate out to the solvent $[25,26]$. Despite the considerable cost of the HHP equipment, processing can become cheaper compared to conventional methods that demand temperature and long processing times [27]. Therefore, the equipment costs could be repaid back in long-term usage.

Previous studies reported that HHP is a good alternative to conventional extraction methods since it avoids the degradation of thermosensitive molecules, reduces the extraction time and solvent consumption, and improves the extraction efficiency in terms of yield, quality and selectivity [24-26,28-30]. Moreover, a low-energy input is required by this eco-friendly technology to compress a sample to $500 \mathrm{MPa}$ as compared to heating to $100^{\circ} \mathrm{C}$ [31]. As examples, HHP was successfully applied to extract antioxidant compounds from pomegranate [32] and citrus [33] peels and fig by-products [30], flavonoids from propolis [29], anthocyanins from grape skins [34], catechins [25] and caffeine [35] from green tea leaves, ginsenosides from ginseng (Panax ginseng C.A. Meyer) [36], ferulic acid from Radix Angelica sinensis [37], and carotenoids from tomato wastes [22]. However, the performance of this extraction method can be affected by a number of independent variables such as processing time, pressure and solvent $[30,32]$, whose effect on one or more dependent (response) variables can be evaluated using the response surface methodology (RSM). This is a time- and reagent-saving statistical tool increasingly used in process optimization since one-factor-at-a-time experiments cannot predict optimal conditions and neglect interactions between variables.

The present study was carried out to optimize the HHP extraction of phenolic compounds from watercress using RSM. The response variables used in the development of mathematical models describing the extraction process (namely individual and grouped phenolic compounds) were obtained by high-performance liquid chromatography coupled to mass spectrometry (HPLC-DAD-ESI/MS).

\section{Material and methods}

\subsection{Standards and reagents}

HPLC-grade acetonitrile was purchased from Fisher Scientific (Lisbon, Portugal). Formic acid was purchased from Prolabo (VWR International, France). The phenolic compound standards (ferulic, sinapic, $p$-coumaric and caffeic acids, and kaempferol-3-O-rutinoside, quercetin-3-O-rutinoside, and quercetin-3-O-glucoside) were purchased from Extrasynthese (Genay, France). All other chemicals were of analytical grade and were purchased from common sources. Water was treated in a Milli-Q water purification system (Millipore, model A10, Billerica, MA, USA).

\subsection{Plant material}

Fresh samples of watercress (Nasturtium officinale R. Br.) were commercially obtained from a local supermarket in Bragança, Portugal. The taxonomic identification of the plant material was confirmed by the botanist Dr. Ana Maria Carvalho from the Polytechnic Institute of Bragança, Portugal. The samples were lyophilized (FreeZone 4.5, Labconco, Kansas City, MO, USA), reduced to a fine powder $(\sim 20$ mesh), and kept at $-20^{\circ} \mathrm{C}$ until processing.

\subsection{High hydrostatic pressure extraction}

The extractions were carried out on a pilot-scale high-pressure equipment (Model 55, Hyperbaric, Burgos, Spain) with a pressure vessel of $55 \mathrm{~L}$, connected to a refrigeration unit (RMA KH $40 \mathrm{LT}$, Ferroli, San Bonifacio, Italy) to control the temperature of the input water used as pressure-transmitting fluid. Heat-sealed plastic bags containing $0.6 \mathrm{~g}$ of dry powder sample and $20 \mathrm{~mL}$ of solvent were placed in the pressure vessel and then subjected to different conditions of processing time $(1.5-33.5 \mathrm{~min})$, pressure $(0.1-600 \mathrm{MPa})$ and solvent $(0-100 \%$ of ethanol, $\mathrm{v} / \mathrm{v}$ ) as defined in the circumscribed central composite design (CCCD) presented in Table 1. Ethanol:water mixtures were used since ethanol has low toxicity and GRAS (generally recognized as safe) status. The solid/liquid ratio was maintained at $30 \mathrm{~g} / \mathrm{L}$. All extractions were performed at $20^{\circ} \mathrm{C}$ (cold extraction). However, since the pressure increases the temperature by $\sim 3{ }^{\circ} \mathrm{C} / 100 \mathrm{MPa}[22,23]$, processing at $600 \mathrm{MPa}$ resulted in an adiabatic temperature increase from $20^{\circ} \mathrm{C}$ to $\sim 38^{\circ} \mathrm{C}$, which should still be not enough to promote the thermal degradation of bioactive compounds. After HHP processing, the mixture was filtered through filter paper (Whatman No. 4) and the filtrate was collected and kept at $-80^{\circ} \mathrm{C}$ until analysis.

\subsection{Calculation of the extraction yield}

The extraction yields (\%) were calculated based on the dry weight (crude extract) obtained after evaporation of the solvent. First, the filtrates were concentrated at $35^{\circ} \mathrm{C}$ under reduced pressure (rotary evaporator Büchi R-210, Flawil, Switzerland) and the aqueous phase was then lyophilised to obtain a dried extract.

\subsection{Chromatographic analysis of phenolic compounds}

The dried extracts $(\sim 10 \mathrm{mg})$ were dissolved in a methanol:water mixture $(20: 80 \mathrm{v} / \mathrm{v})$ and filtered through $0.22 \mu \mathrm{m}$ disposable LC filter disks. The chromatographic analysis was performed in a Dionex Ultimate 3000 UPLC (Thermo Scientific, San Jose, CA, USA) system equipped with a diode array detector (DAD) coupled to an electrospray ionization mass detector (ESI-MS) (ThermoFinnigan, San Jose, CA, USA) as described by Bessada et al. [38]. The phenolic compounds were identified using $280 \mathrm{~nm}$ and $370 \mathrm{~nm}$ as preferred wavelengths and by comparing their retention time and UV-vis and mass spectra with those obtained from authentic standards, when available. For quantitative analysis, a baseline to valley integration with baseline projection mode was used to calculate the peak areas and the external standards mentioned above were used for quantification. The results were expressed in $\mathrm{mg}$ per $\mathrm{g}$ of extract.

\subsection{Experimental design, modelling and optimization}

\subsubsection{Experimental design}

A five-level CCCD (Box-Behnken design) coupled with RSM was implemented to optimize the HHP conditions for the extraction of phenolic compounds from watercress. The coded and natural values of the independent variables $X_{1}$ (processing time $(t), \min$ ), $X_{2}$ (pressure $(P), \mathrm{MPa}$ ) and $\mathrm{X}_{3}$ (solvent $(S), \%$ of ethanol, $\mathrm{v} / \mathrm{v}$ ) are presented in Table 1. This CCCD includes 6 replicated center points and a group of axial points chosen to allow rotatability, which ensures that the variance of the model prediction is constant at all points equidistant from the design center. The experimental runs were randomized to minimize the effects of unexpected variability in the observed responses.

\subsubsection{Mathematical modelling}

The response surface models were fitted by means of least-squares calculation using the following Box-Behnken design equation: 


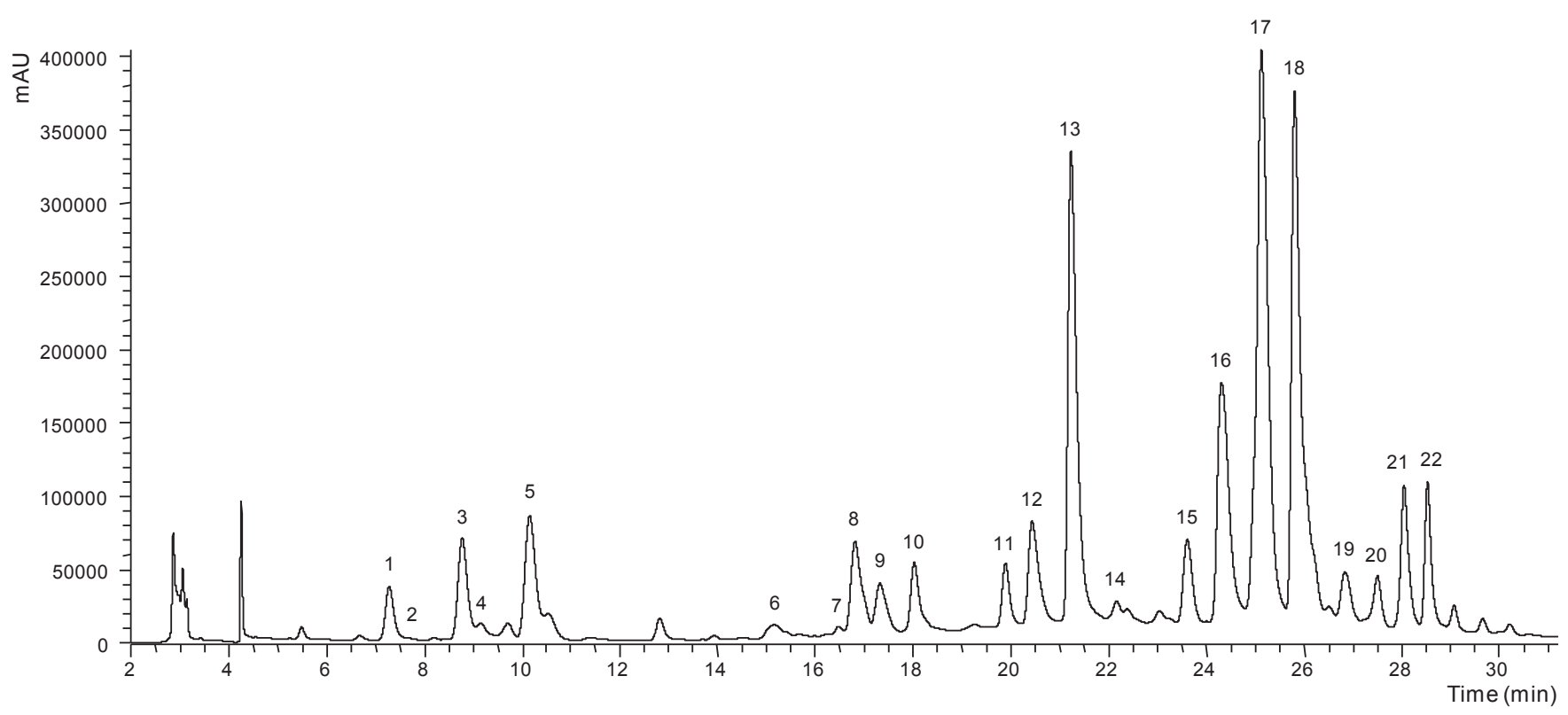

Fig. 1. HPLC profile of phenolic compounds of the watercress extract obtained under the experimental run No. 20 , recorded at 370 nm. See Table 1 for peak identification.

$Y=b_{0}+\sum_{i=1}^{n} b_{i} X_{i}+\sum_{\substack{i=1 \\ j>i}}^{n-1} \sum_{j=2}^{n} b_{i j} X_{i} X_{j}+\sum_{i=1}^{n} b_{i i} X_{i}^{2}$

In this equation, $Y$ represents the dependent variable (response variable) to be modelled, $X_{i}$ and $X_{j}$ are the independent variables, $b_{o}$ is the constant coefficient, $b_{i}$ is the coefficient of linear effect, $b_{i j}$ is the coefficient of interaction effect, $b_{i i}$ is the coefficient of quadratic effect, and $n$ is the number of variables. The extraction yield and the individual and grouped phenolic compounds (22 compounds and 6 groups) were used as dependent variables.

\subsubsection{Procedure to optimize the variables to a maximum response}

A simplex method was used to optimize the predictive model by solving nonlinear problems in order to maximize the extraction yield and the recovery of phenolic compounds [5]. Certain limitations were imposed (i.e., times lower than 0 ) to avoid variables with unnatural and unrealistic physical conditions.

\subsection{Cluster analyses}

A cluster analysis was performed to group the phenolic compounds according to the extraction conditions that maximize their response values using the "XLSTAT 2016", a Microsoft Excel add-in. A comparative agglomerative hierarchical clustering analysis (HCA) with Pearson correlation coefficient was used for clustering (similarity analysis). The algorithm used was a complete linkage with automatic truncation based on entropy.

\subsection{Fitting procedures and statistical analysis}

Fitting procedures, coefficient estimates and statistical calculations were performed as previously described by Pinela et al. [5]. In brief, a) the coefficient measurement was performed using the nonlinear leastsquare (quasi-Newton) method provided by the macro "Solver" in Microsoft Excel, which allows minimizing the sum of the quadratic differences between the observed and model-predicted values; b) the coefficient significance was evaluated using the 'SolverAid' to determine the parametric confidence intervals. The not statistically significant terms ( $p$-value $>0.05$ ) were dropped to simplify the model; and c) the model reliability was verified using the following criteria: i) the Fisher $F$-test $(\alpha=0.05)$ was used to determine whether the constructed models were adequate to describe the observed data; ii) the 'SolverStat' macro was used for the assessment of parameter and model prediction uncertainties; iii) the $\mathrm{R}^{2}$ was interpreted as the proportion of variability of the dependent variable explained by the model.

\section{Results and discussion}

\subsection{Response criteria for the RSM analysis}

The experimental values achieved for the 20 experimental runs of the CCCD design are presented in Table 1. The HPLC phenolic profile (recorded at $370 \mathrm{~nm}$ ) of the watercress extract obtained under the experimental run No. 20 is shown in Fig. 1 (see HHP extraction conditions in Table 1). This profile is concordant with that previously characterized by Pinela et al. [14] for wild watercress. Up to twenty-two compounds were identified (Table 1) based on their chromatographic, UV-vis and mass spectra characteristics, six of which were phenolic acid derivatives (hydroxycinnamic acids) and sixteen were flavonoid glycoside derivatives. Many of these compounds were also reported by other authors in this species $[12,39,40]$. Isorhamnetin-O-hydroxyferuloylhexoside- $O$-malonylhexoside, $p$-coumaric acid, isorhamnetin$O$-sophoroside- $O$-malonylhexoside, quercetin- $O$-sophoroside- $O$-malonylhexoside and caffeic acid were identified as the most abundant compounds. Flavonoids predominated over phenolic acids and, in general, more quercetin and isorhamnetin glycoside derivatives were quantified than phenolic acids.

For optimization purposes, the phenolic compounds quantified by chromatographic methods (Table 1) were grouped in total phenolic acids (compounds 2, 4, 5, 6, 8 and 9), total flavonoids, comprising the subgroups of quercetin glycoside derivatives (Qgd: compounds 1, 3, 7, 10, 11, 12, 13, 14 and 15), isorhamnetin glycoside derivatives (Igd: compounds 16, 17 and 18) and kaempferol glycoside derivatives (Kgd: compounds 19, 20, 21 and 22), and total phenolic compounds (including all quantified phenolics). The individual and grouped compounds were used as response criteria to optimize the HHP conditions for their extraction from watercress using RSM. The values of the extraction yield were also considered, which ranged from 12 to $27.5 \%$ with the experimental runs $n^{\circ} 14$ and 3 , respectively (Table 1 ). Therefore, a total of 29 response variables were computed and used as optimization criteria. 


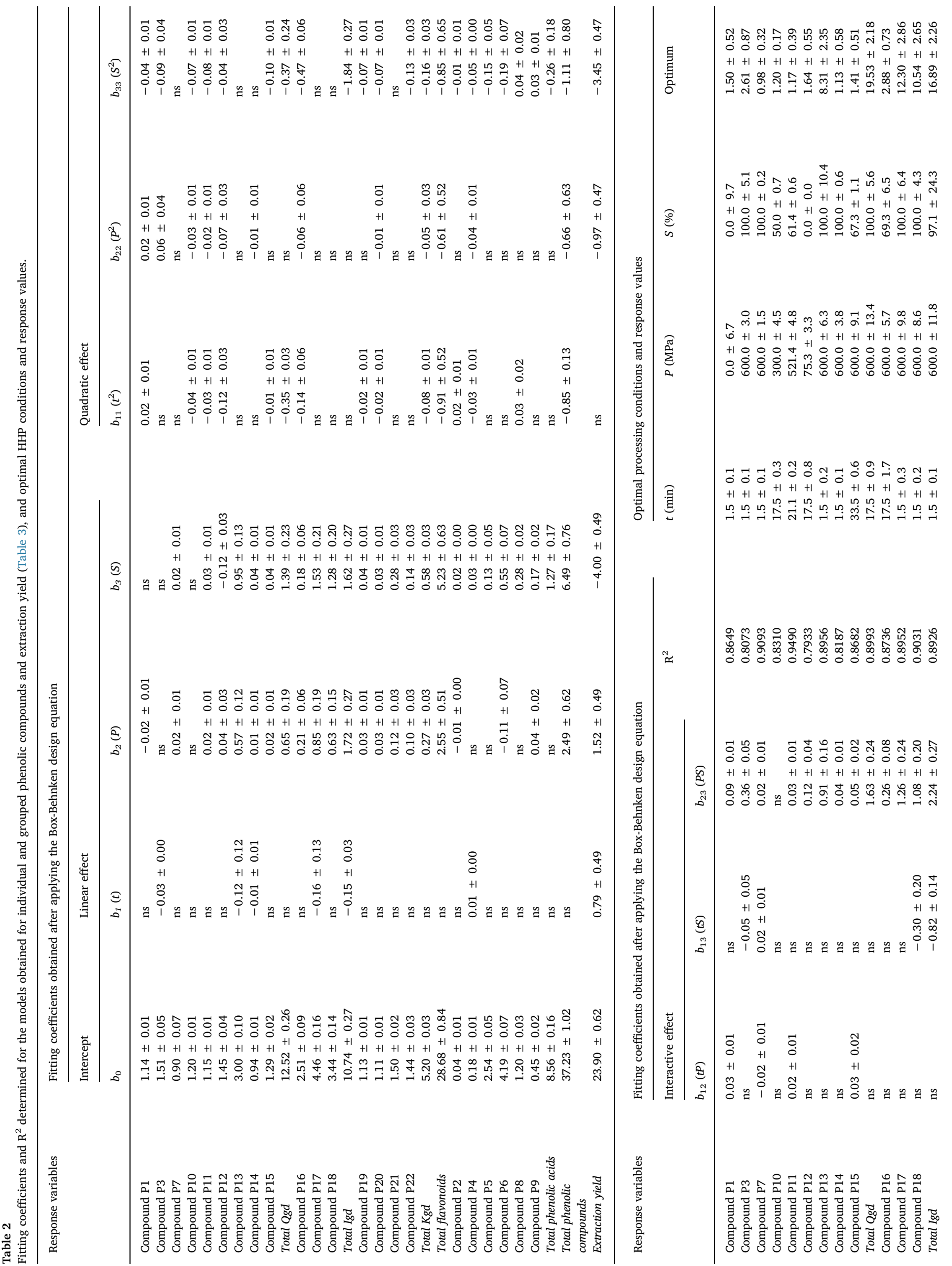




\subsection{Theoretical response surface models}

As in many research fields, when trying to develop theoretical models to predict and comprehend the effects of independent variables on certain response variables, it is necessary to evaluate its precision by fitting these models to the experimental values. In this study, the response values (Table 1) were fitted to a second-order polynomial model using a nonlinear algorithm (least-squares estimations) to develop mathematical models for each response criteria (Table 2). Table 3 shows the estimated coefficient values obtained from the polynomial model of Eq. (1) and the coefficient of correlation $\left(R^{2}\right)$ for each parametric response of the extraction process. These parametric values translate the response patterns and show the complexity of the possible interactions between variables. However, not all the parameters of Eq. (1) were used for building the model since some coefficients were nonsignificant (ns). The significant ones were assessed at a $95 \%$ confidence level $(\alpha=0.05)$. The statistic lack of fit, used to test the adequacy of the obtained models, demonstrated that no considerable improvement was achieved by the inclusion of the statistically $n s$ parametric values. The resulting models for each of the 29 assessed responses are presented in Table 2. In all cases, $\mathrm{R}^{2}$ coefficients higher than 0.79 were obtained (Table 3), which indicates that the percentage of variability of each response can be explained by the model. These workable models were applied in the subsequent prediction and optimization steps, showing a good agreement between the experimental and predicted values, which indicates that the variation is explained by the independent variables.

The obtained model coefficients (Table 3) are empirical and cannot be associated with physical or chemical significance. However, they are useful for predicting the results of untested extraction conditions [41]. The sign of the effect marks the performance of the response. In this way, when a factor has a positive effect, the response is higher at the high level, and when a factor has a negative effect, the response is lower at the high level. The higher the absolute value of a coefficient, the more important the weight of the corresponding variable. Based on the mathematical expressions (Table 2), no associations were found between the response variables of phenolic acids, flavonoids, quercetin glycoside derivatives $(Q g d)$, isorhamnetin glycoside derivatives (Igd) and kaempferol glycoside derivatives $(\mathrm{Kgd})$. However, certain features regarding the general effects of the variables are displayed. The relevance of the significant parametric values can be order as a function of the variables involved in a decreasing form as $S>P \gg t$. Alexandre et al. [32] also found $S$ as the most relevant variable on the HHP extraction of bioactive compounds from pomegranate (Punica granatum L.) peels. Regarding the linear, quadratic, and interactive parametric effects of the developed equations, it was found that they play an important and significant role in all evaluated responses. For the linear effect, the variables $P$ and $S$ had strong values; meanwhile, the effect of $t$ was negligible in almost all cases. All independent variables had moderate quadratic or nonlinear effects. Regarding the interactive effects, the interactions of the variable $t$ with the other variables ( $t P$ and $t S$ ) were of minor relevance; meanwhile, the $P S$ interaction had a strong significance in describing the behavior of almost all responses (with the exception of compound 10). The interactive parametric values of $P S$ were accentuated in the responses of flavonoids, Qgd, Igd, phenolic acids, and total phenolic compounds. To make the combined effects more explicit and to visually describe the extraction trends, the results were presented in the response surface plots discussed below.

\subsection{Effect of the independent variables on the target responses and optimal extraction conditions}

Although parametric results can depict the patterns of the responses, 3D and 2D graphical representations may aid on their comprehension. Fig. 2 shows the response surface plots of extraction yield and grouped phenolic compounds (total phenolic acids, total flavonoids 
Table 3

Mathematical models of the extraction process derived from the second-order polynomial model with interactions of Eq. (1).

\begin{tabular}{|c|c|c|}
\hline Quercetin-3-O-sophoroside & $Y_{P 1}=1.14-0.02 P+0.02 t^{2}+0.02 P^{2}-0.04 S^{2}+0.09 t P+0.09 P S$ & Eq. (2) \\
\hline Quercetin-3-O-manolylglucoside-7-O-glucoside & $Y_{P 3}=1.51-0.03 t+0.06 P^{2}-0.09 S^{2}-0.05 t S+0.36 P S$ & Eq. (3) \\
\hline Quercetin-3-O-rutinoside-7-O-glucoside & $Y_{P 7}=0.90+0.02 P+0.02 S^{2}-0.02 t P+0.02 t S+0.02 P S$ & Eq. (4) \\
\hline Quercetin-3-O-rutinoside (rutin) & $Y_{P 10}=1.20-0.04 t^{2}-0.03 P^{2}-0.07 S^{2}$ & Eq. (5) \\
\hline Quercetin-O-sophoroside- $O$-rutinoside & $Y_{P 11}=1.15+0.02 P+0.03 S-0.03 t^{2}-0.23 P^{2}-0.02 S^{2}-0.08 t P+0.02 t S$ & Eq. (6) \\
\hline Quercetin-O-coumaroylsophoroside & $Y_{P 12}=1.45+0.04 P-0.12 S-0.12 t^{2}-0.07 P^{2}-0.04 S^{2}+0.12 P S$ & Eq. (7) \\
\hline Quercetin-O-sophoroside-O-malonylhexoside & $Y_{P 13}=3.00-0.12 t+0.57 P+0.95 S$ & Eq. (8) \\
\hline Quercetin-O-dihexosyl-O-malonylhexoside & $Y_{P 14}=0.94-0.01 t+0.01 P+0.04 S-0.01 P^{2}+0.04 P S$ & Eq. (9) \\
\hline Quercetin-O-sinapoylhexoside-O-rutinoside & $Y_{P 15}=1.29+0.02 P+0.04 S-0.01 t^{2}-0.10 S^{2}-0.03 t P+0.05 P S$ & Eq. (10) \\
\hline Total quercetin glycoside derivatives ( $Q g d$ ) & $Y_{Q g d}=12.52+0.65 P+1.39 S-0.35 t^{2}-0.37 S^{2}+1.63 P S$ & Eq. (11) \\
\hline Isorhamnetin-O-hydroxyferuloylhexoside-O-hexoside & $Y_{P 16}=2.51+0.21 P+0.18 S-0.14 t^{2}-0.06 P^{2}-0.47 S^{2}+0.26 P S$ & Eq. (12) \\
\hline Isorhamnetin-O-hydroxyferuloylhexoside-O-malonylhexoside & $Y_{P 17}=4.46-0.16 t+0.85 P+1.53 S+1.26 P S$ & Eq. (13) \\
\hline Isorhamnetin-O-sophoroside-O-malonylhexoside & $Y_{P 18}=3.44+0.63 P+1.28 S-0.30 t S+1.08 P S$ & Eq. (14) \\
\hline Total isorhamnetin glycoside derivatives (Igd) & $Y_{I g d}=10.74-0.15 t+1.72 P+1.62 S-1.84 S^{2}-0.82 t S+2.24 P S$ & Eq. (15) \\
\hline Kaempferol-O-feruloylhexoside-O-rutinoside & $Y_{P 19}=1.13+0.03 P+0.04 S-0.02 t^{2}-0.07 S^{2}+0.02 t P+0.05 P S$ & Eq. (16) \\
\hline Kaempferol-O-feruloylhexoside-O-hexoside & $Y_{P 20}=1.11+0.03 P+0.03 S-0.02 t^{2}-0.01 P^{2}-0.07 S^{2}+0.02 t P+0.04 P S$ & Eq. (17) \\
\hline Kaempferol-O-hydroxyferuloylglucuronide-O-malonylhexoside & $Y_{P 21}=1.50+0.12 P+0.28 S-0.05 t S+0.23 P S$ & Eq. (18) \\
\hline Kaempferol-O-feruloylhexoside-O-malonylhexoside & $Y_{P 22}=1.44+0.10 P+0.14 S-0.13 S^{2}-0.04 t S+0.19 P S$ & Eq. (19) \\
\hline Total kaempferol glycoside derivatives (Kgd) & $Y_{K g d}=5.20+0.27 P+0.58 S-0.08 t^{2}-0.05 P^{2}-0.16 S^{2}+0.07 t P-0.11 t S+0.51 P S$ & Eq. (20) \\
\hline Total flavonoids & $Y_{F l}=28.68+2.55 P+5.23 S-0.91 t^{2}-0.61 P^{2}-0.85 S^{2}-1.11 t S+4.90 P S$ & Eq. (21) \\
\hline$p$-Coumaric acid hexoside & $Y_{P 2}=0.04-0.01 P+0.02 S+0.02 t^{2}-0.01 S^{2}-0.02 P S$ & Eq. (22) \\
\hline Ferulic acid hexoside & $Y_{P 4}=0.18+0.01 t+0.03 S-0.03 t^{2}-0.04 P^{2}-0.05 S^{2}+0.02 t P-0.01 t S-0.01 P S$ & Eq. (23) \\
\hline Caffeic acid & $Y_{P 5}=2.54+0.13 S-015 S^{2}+0.08 t P-0.12 t S+0.47 P S$ & Eq. (24) \\
\hline$p$-Coumaric acid & $Y_{P 6}=4.19-0.11 P+0.55 S-0.19 S^{2}+0.23 t P-0.16 t S+0.45 P S$ & Eq. (25) \\
\hline Ferulic acid & $Y_{P 8}=1.20+0.28 S-0.03 t^{2}-0.04 S^{2}-0.03 t P-0.07 t S+0.19 P S$ & Eq. (26) \\
\hline Sinapoylmalic acid & $Y_{P 9}=0.45+0.04 P+0.17 S-0.03 S^{2}-0.01 t S+0.10 P S$ & Eq. (27) \\
\hline Total phenolic acids & $Y_{P a}=8.56+1.27 S-0.26 S^{2}+0.39 t P-0.42 t S+1.25 P S$ & Eq. (28) \\
\hline Total phenolic compounds & $Y_{P h}=37.23+2.49 P+6.49 S-0.85 t^{2}-0.66 P^{2}-1.11 S^{2}-1.53 t S+6.15 P S$ & Eq. (29) \\
\hline Extraction yield (crude extract) & $Y_{E Y}=23.90+0.79 t+1.52 P-4.01 S-0.97 P^{2}-3.45 S^{2}+1.21 P S$ & Eq. (30) \\
\hline
\end{tabular}

and total phenolic compounds) as well as their statistical analysis. Fig. 3 illustrates in a similar way the results for Qgd, Igs and Kgd. Both Figs. 2 and 3 are divided in three subsections: i) the subsection A illustrates the $3 \mathrm{D}$ response surface plots, whose grid surfaces were predicted with the respective second-order polynomial model described by Eq. (1) using the theoretical values presented in Table 3. For representation of these binary combinations, the excluded variable was positioned at the optimum of their experimental domain (Table 3); ii) the subsection B illustrates the goodness of fit through two graphical statistical criteria, namely the ability to simulate response changes between observed and predicted values and the residual distribution as a function of each variable; and iii) the subsection $C$ shows the individual $2 \mathrm{D}$ responses and the optimum values $(\odot)$. In each plot, each independent variable was positioned at the optimal value of the other two variables.

Observing the response surface plots of the extraction yield (Fig. 2), it is possible to verify that the amount of extracted material increases to an optimum value and then, in most cases, it decreases as a function of the involved independent variable. Consequently, the optimum value can be found as being a single point in almost all combinations, which allows computing the extraction conditions that lead to an absolute maximum. Fig. 2C simplifies the interpretation of the effects of the independent variables on the extraction process and highlights the optimum value of each variable. The extraction yield was maximal $(27.82 \pm 2.26 \%)$ when the optimal HHP conditions $(t=33.5 \mathrm{~min}$, $P=530.6 \mathrm{MPa}$ and $S=26.1 \%$ of ethanol, v/v) presented in Table 3 were applied for extraction. Zhang et al. [42] have shown that the crude extract obtained from Rhodiola sachalinensis is greater when HHP is used than when the extraction is done by the conventional methods of reflux or Soxhlet. High extraction yields were also achieved by Prasad et al. [26] when processing longan fruit (Dimpcarpus longan Lour.) pericarps under pressures up to $500 \mathrm{MPa}$ and using lower extraction times than those required in a conventional extraction.

The response surface plots of grouped phenolic acids and flavonoids and total phenolic compounds are showed in Fig. 2 and the optimal
HHP conditions that maximize their recovery from watercress are presented in Table 3 . These responses were similarly affected by the screened variables; they were favoured by high values of $P$ and $S$ and short values of $t$ as summarized below:

- For phenolic acids, the optimal HHP conditions were: $t=1.5 \pm 0.3 \mathrm{~min}, P=600.0 \pm 5.0 \mathrm{MPa}$ and $S=100.0 \pm 8.6 \%$ of ethanol $(\mathrm{v} / \mathrm{v})$, and originated $13.58 \pm 1.99 \mathrm{mg} / \mathrm{g}$ of extract.

- For flavonoids, the optimal HHP conditions were: $t=7.8 \pm 0.5 \mathrm{~min}, P=600.0 \pm 5.0 \mathrm{MPa}$ and $S=100.0 \pm 8.6 \%$ of ethanol $(\mathrm{v} / \mathrm{v})$, and originated $52.45 \pm 2.63 \mathrm{mg} / \mathrm{g}$ of extract.

- For total phenolic compounds, the optimal HHP conditions were: $t=3.1 \pm 3.2 \mathrm{~min}, P=600.0 \pm 6.3 \mathrm{MPa}$ and $S=100.0 \pm 2.2$ of ethanol $(\mathrm{v} / \mathrm{v})$, and originated $64.68 \pm 2.97 \mathrm{mg} / \mathrm{g}$ of extract.

The optimum extraction values for the flavonoid derivatives $Q g d$, Igs and $K g d$ were achieved using very similar HHP conditions (Fig. 3 and Table 3), probably due to structural similarities between these compounds. Once more, the extraction was favoured by high values of $P$ and $S$ and short values of $t$, as summarized below:

- For Qgd, the optimal HHP conditions were: $t=17.5 \pm 1.0 \mathrm{~min}$, $P=600.0 \pm 13.4 \mathrm{MPa}$ and $100.0 \pm 5.6 \%$ of ethanol $(\mathrm{v} / \mathrm{v})$, and originated $19.53 \pm 2.18 \mathrm{mg} / \mathrm{g}$ of extract.

- For Igd, the optimal HHP conditions were: $t=1.5 \pm 0.1 \mathrm{~min}$, $P=600.0 \pm 11.8 \mathrm{MPa}$ and $97.1 \pm 24.3 \%$ of ethanol, and originated $16.89 \pm 2.26 \mathrm{mg} / \mathrm{g}$ of extract.

- For Qgd, the optimal HHP conditions were: $t=13.7 \pm 0.4 \mathrm{~min}$, $P=600.0 \pm 16.4 \mathrm{MPa}$ and $S=100.0 \pm 2.7 \%$ of ethanol $(\mathrm{v} / \mathrm{v})$, and originated $7.49 \pm 0.88 \mathrm{mg} / \mathrm{g}$ of extract.

According to the literature, the use of high pressures increases the extraction of bioactive compounds from plants matrices [30]. BrionesLabarca et al. [28] demonstrated that HHP is more effective than 


\section{EXTRACTION YIELD}

A: JOINT ANALYSIS IN 3D
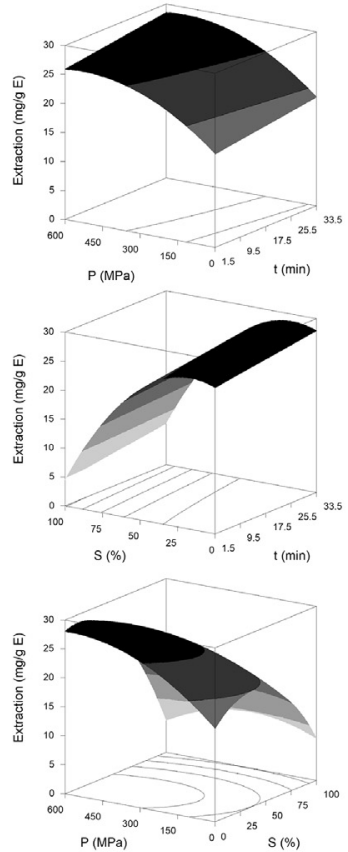

B: STATISTICAL DISTRIBUTION

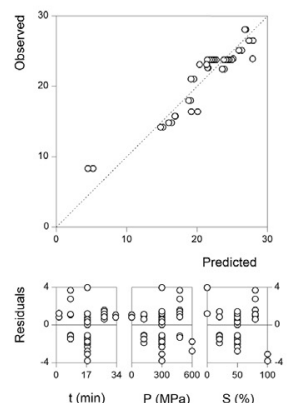

C: ILLUSTRATION IN 2D

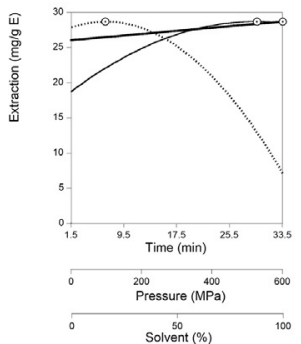

PHENOLIC ACIDS

A: JOINT ANALYSIS IN 3D

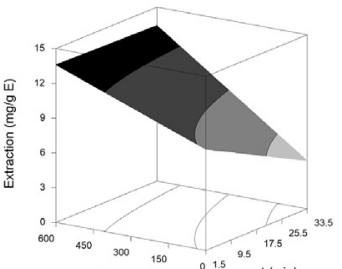

$P(\mathrm{MPa}) \quad 01.5 \mathrm{t}(\mathrm{min})$
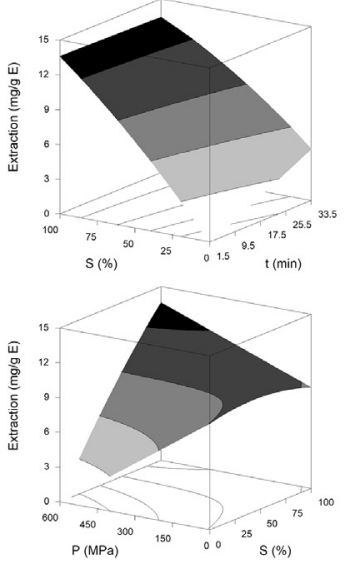

B: STATISTICAL DISTRIBUTION

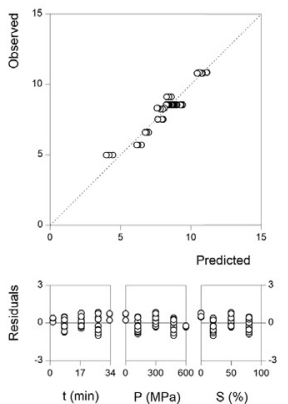

C: ILLUSTRATION IN 2D

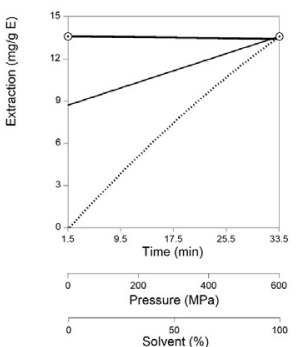

FLAVONOIDS

A: JOINT ANALYSIS IN 3D
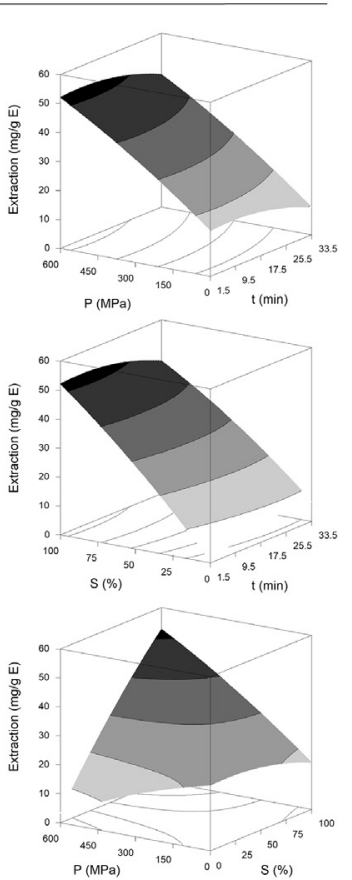

B: STATISTICAL DISTRIBUTION
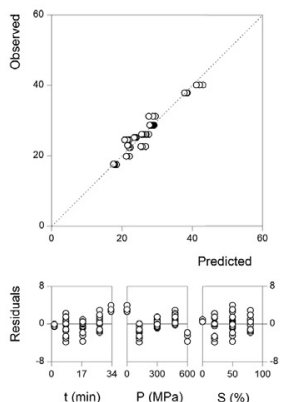

C: ILLUSTRATION IN 2D

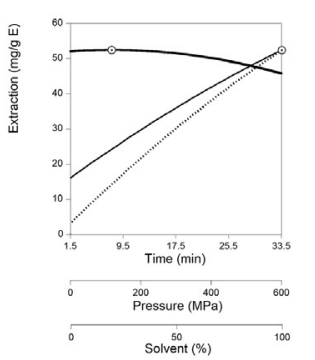

TOTAL PHENOLICS

A: JOINT ANALYSIS IN 3D
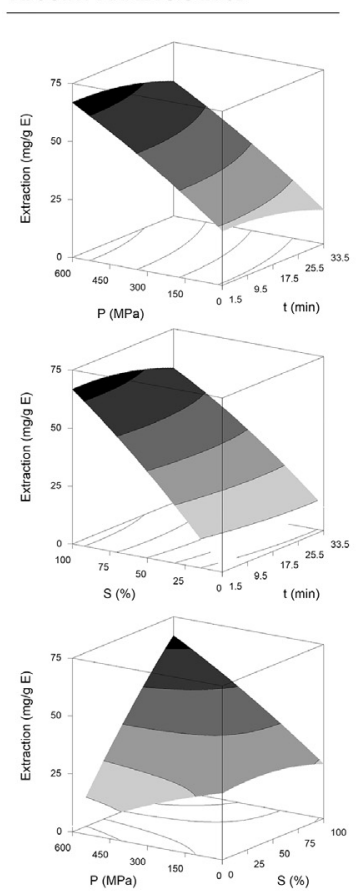

B: STATISTICAL DISTRIBUTION

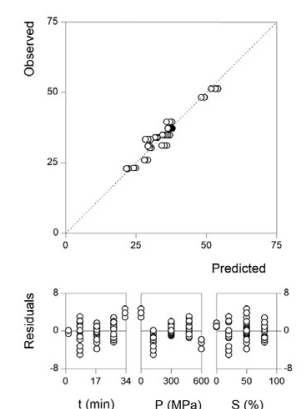

C: ILLUSTRATION IN 2D

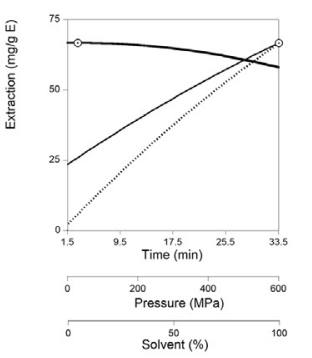

$-\quad$ Time $(\min ) \quad$ Pressure $(\mathrm{MPa}) \quad \ldots . . . . . . . \quad$ Solvent $(\%)$

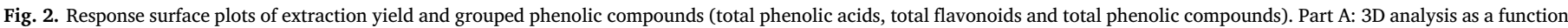

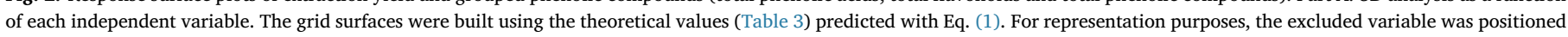

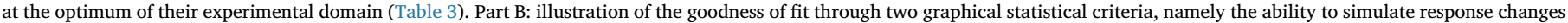

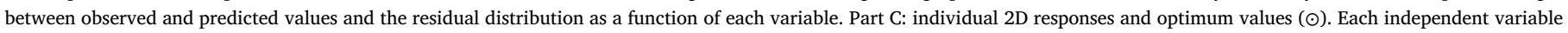
was positioned at the optimal value of the other two variables.

ultrasound-assisted extraction or conventional extraction $(2 \mathrm{~h})$ to recover antioxidants and total phenolic compounds from Chilean papaya (Vasconcellea pubescens) seeds. In addition, HHP was a time-saving extraction method. The lower energy consumption is another advantage of HHP comparatively to conventional methods [35]. In our study, it is also interesting to note that the HHP conditions that maximize the yield of crude extract and the recovery of phenolic compounds differ mostly in the required processing time and ethanol concentration. In this way, the extracts obtained under the optimal conditions established for phenolic compounds (Table 3 ) will contain a lower quantity of compounds other than phenolics, thus making the recovery process more selective for the target compounds. 


\section{QUERCETIN DERIVATIVES}

A: JOINT ANALYSIS IN 3D
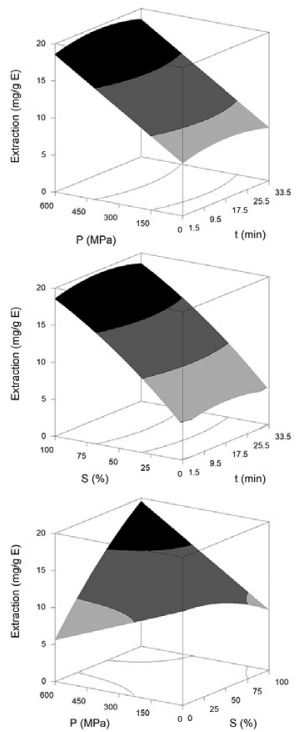

B: STATISTICAL DISTRIBUTION

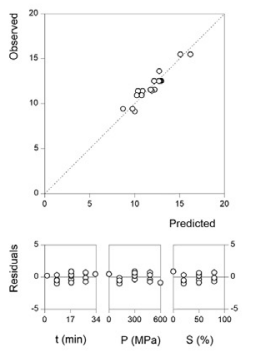

C: ILLUSTRATION IN 2D

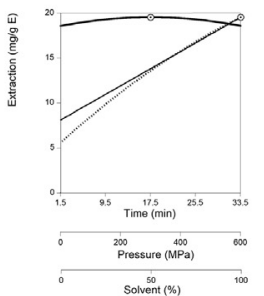

A: JOINT ANALYSIS IN 3D

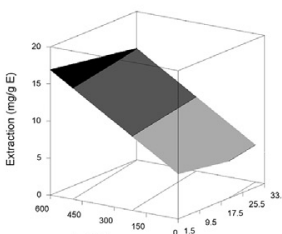

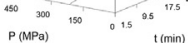
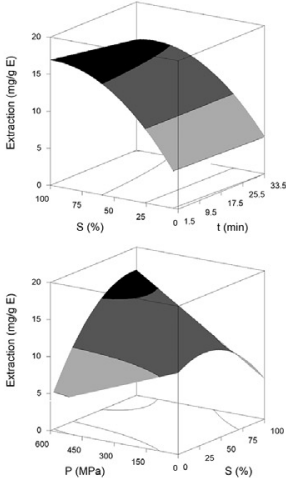

B: STATISTICAL DISTRIBUTION

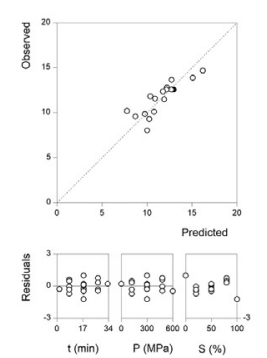

C: ILLUSTRATION IN 2D

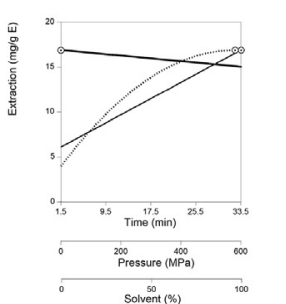

\section{KAEMPFEROL DERIVATIVES}

A: JOINT ANALYSIS IN 3D
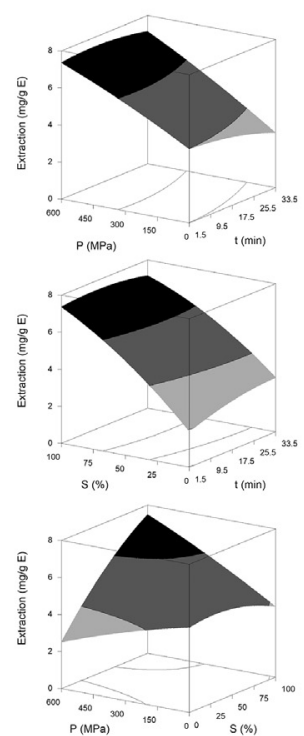

B: STATISTICAL DISTRIBUTION

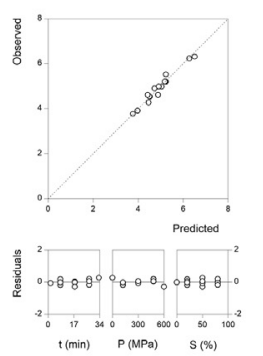

C: ILLUSTRATION IN 2D

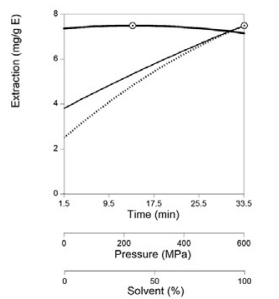

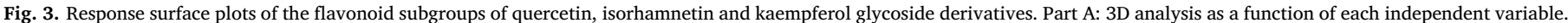

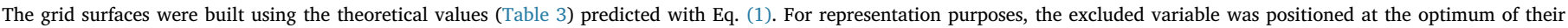

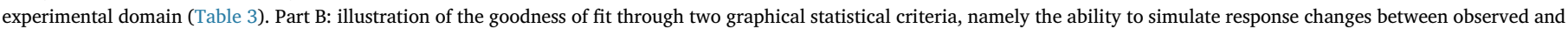

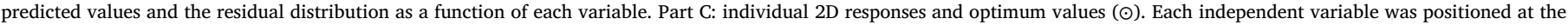
optimal value of the other two variables.

The effects of the independent variables on the extraction of individual phenolic compounds from watercress are 2D represented in Fig. 4. The processing conditions that generated optimal response values $(\odot)$ are numerically described in Table 3 . The identified flavonoids were organized as a function of the maximum amount achieved $(\mathrm{mg} / \mathrm{g}$ of extract) in a decreasing order as follows: P17 $(12.3 \pm 2.86)>$ P18 $(10.54 \pm 2.65)>\mathrm{P} 13(8.31 \pm 2.35) \gg \mathrm{P} 21(2.95 \pm 1.40)>\mathrm{P} 16$ $(2.88 \pm 0.73)>$ P3 $(2.61 \pm 0.87)>$ P22 $(2.11 \pm 0.79)>$ P12 $(1.64 \pm 0.55)>\mathrm{P} 1 \quad(1.5 \pm 0.52)>\mathrm{P} 15 \quad(1.41 \pm 0.51)>\mathrm{P} 19$ $(1.26 \pm 0.48)>\mathrm{P} 10 \quad(1.2 \pm 0.17)>\mathrm{P} 11 \quad(1.17 \pm 0.39)>\mathrm{P} 20$ $(1.16 \pm 0.39)>$ P14 $(1.13 \pm 0.58)>$ P7 $(0.98 \pm 0.32)$. Meanwhile, the phenolic acids were organized as follows: P6 $(5.02 \pm 0.96)>\mathrm{P} 5 \quad(3.79 \pm 0.60) \gg \mathrm{P} 8 \quad(2.51 \pm 0.68)>\mathrm{P} 9$ $(1.21 \pm 0.60)>\mathrm{P} 4(0.19 \pm 0.14)>\mathrm{P} 2(0.06 \pm 0.08)$. Pinela et al.
[14] reported lower quantities of phenolic acids $(5.6 \pm 0.5 \mathrm{mg} / \mathrm{g}$ of extract), flavonoids (22 $\pm 1 \mathrm{mg} / \mathrm{g}$ of extract) and total phenolic compounds $(28 \pm 2 \mathrm{mg} / \mathrm{g}$ of extract) in an extract of wild watercress obtained by a conventional solid-liquid extraction of $2 \mathrm{~h}$ and using a methanol:water mixture (80:20, v/v) as a extraction solvent. These differences highlight the suitability of HHP as an innovative extraction technique to recover a greater amount of phenolic compounds from watercress using shorter processing times and greener solvents.

3.4. Clustering of phenolic compounds according to the HHP conditions that maximize their extraction

Table 4 shows the maximum response values of each phenolic compound and their values if extracted under the optimal HHP 


\section{FLAVONOIDS}
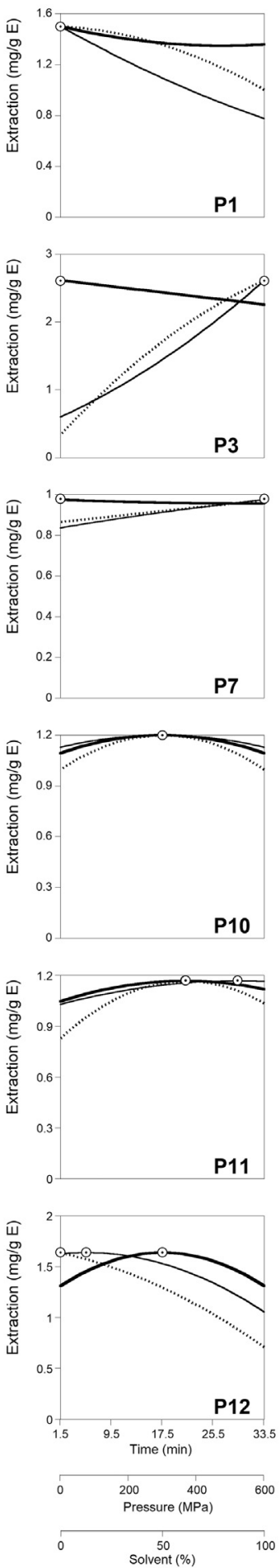
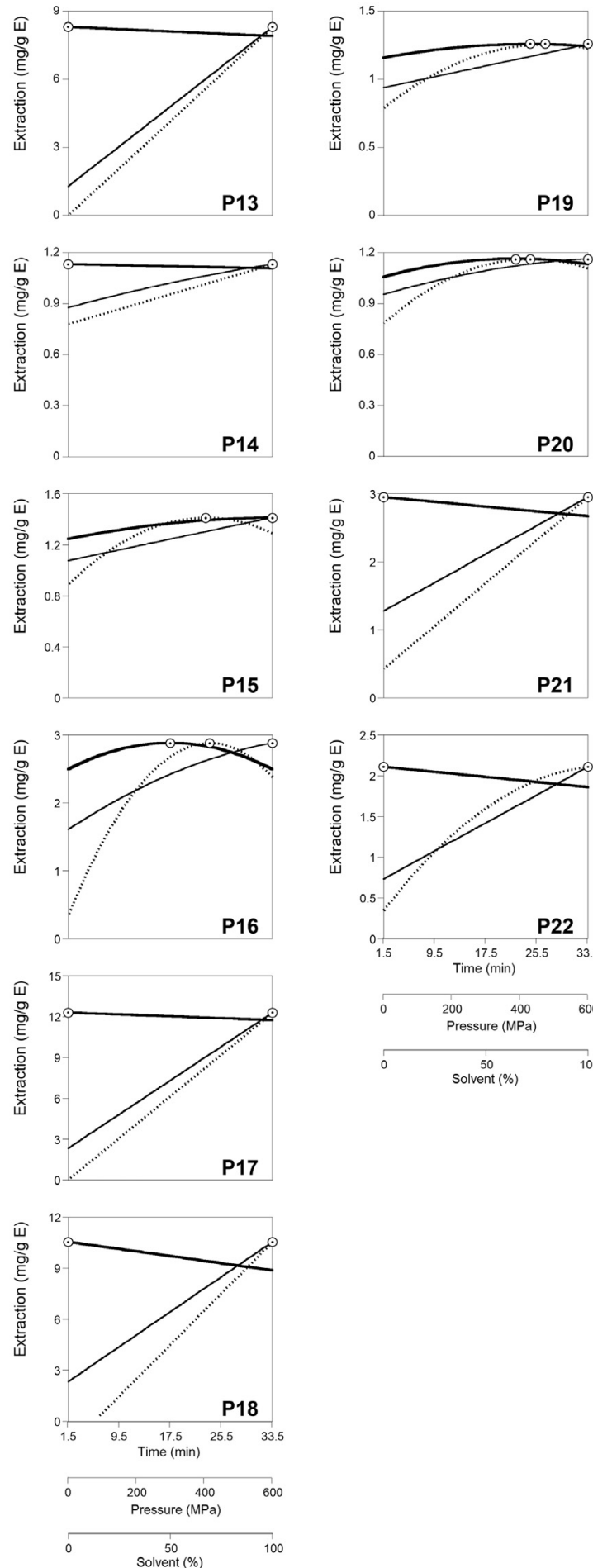

\section{PHENOLIC ACIDS}
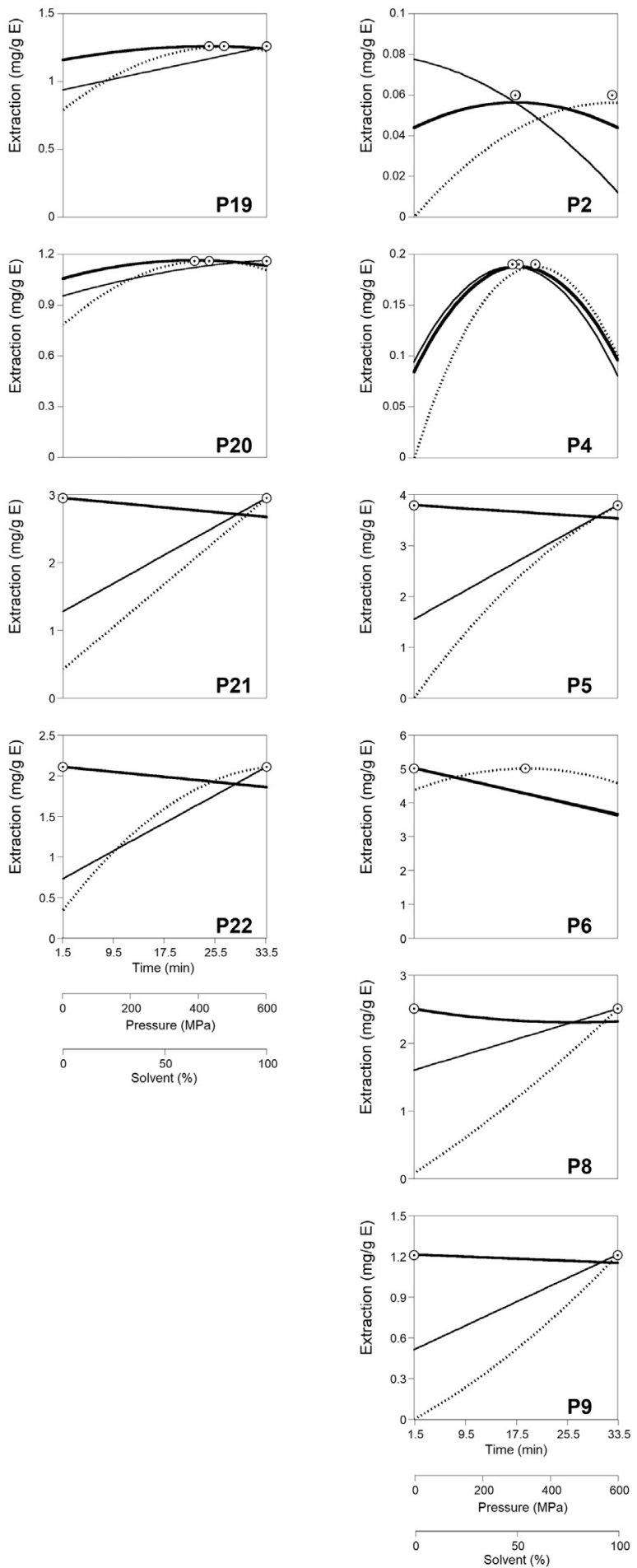

Time (min)

Pressure (MPa) ……... Solvent (\%)

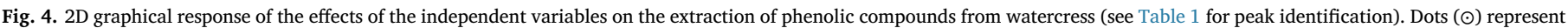
the optimal values. In each plot, each independent variable was positioned at the optimal value of the other two variables (Table 3)

conditions of the other compounds (Table 3). These values presented in part B were calculated dividing the optimum value of each compound by the maximum of the others compounds. Therefore, when two compounds display the value 1 (corresponding to values of $100 \%$ ), the optimum response value of both compounds is achieved under the same HHP conditions. This is the case of compounds $3,5,7,8,9,13,14,17$, 18, 21 and 22, which were clustered in C3a under the same HHP conditions (Fig. 5). In turn, when a 0 is display, it means that the 
Table 4

Maximum response values for each phenolic compound and their values at the optimal processing conditions of the other compounds presented in Table 2 .

A) Maximum response values (mg/g of extract) for the individual phenolic compounds

\begin{tabular}{|c|c|c|c|c|c|c|c|c|c|c|c|c|c|c|c|c|c|c|c|c|c|c|}
\hline Peak: & P1 & P2 & P3 & P4 & P5 & P6 & P7 & P8 & P9 & P10 & P11 & P12 & P13 & P14 & P15 & P16 & P17 & P18 & P19 & P20 & P21 & P22 \\
\hline Optimum: & 1.50 & 0.06 & 2.61 & 0.19 & 3.79 & 5.02 & 0.98 & 2.51 & 1.21 & 1.20 & 1.17 & 1.64 & 8.31 & 1.13 & 1.41 & 2.88 & 12.30 & 10.54 & 1.26 & 1.16 & 2.95 & 2.11 \\
\hline \multicolumn{23}{|c|}{ B) Values for each phenolic compound (\%) at the optimal conditions of the other compounds } \\
\hline & P1 & $\mathrm{P} 2$ & P3 & P4 & P5 & P6 & P7 & P8 & P9 & $\mathrm{P} 10$ & P11 & P12 & P13 & P14 & P15 & P16 & P17 & P18 & P19 & P20 & P21 & P22 \\
\hline P1 & 1 & 0.69 & 0.85 & 0.75 & 0.85 & 0.89 & 0.85 & 0.85 & 0.85 & 0.76 & 0.79 & 0.85 & 0.85 & 0.85 & 0.90 & 0.81 & 0.85 & 0.85 & 0.86 & 0.84 & 0.85 & 0.85 \\
\hline P2 & 0 & 1 & 0 & 0.86 & 0 & 0.59 & 0 & 0 & 0 & 0.76 & 0.54 & 0 & 0 & 0 & 0.16 & 0.38 & 0 & 0 & 0.30 & 0.35 & 0 & 0 \\
\hline P3 & 0.90 & 0.49 & 1 & 0.57 & 1 & 0.62 & 1 & 1 & 1 & 0.58 & 0.66 & 0.81 & 1 & 1 & 0.73 & 0.77 & 1 & 1 & 0.81 & 0.78 & 1 & 1 \\
\hline P4 & 0 & 0.59 & 0 & 1 & 0 & 0.05 & 0 & 0 & 0 & 0.97 & 0.67 & 0 & 0 & 0 & 0.01 & 0.35 & 0 & 0 & 0.19 & 0.33 & 0 & 0 \\
\hline P5 & 0.82 & 0.62 & 1 & 0.67 & 1 & 0.71 & 1 & 1 & 1 & 0.67 & 0.74 & 0.77 & 1 & 1 & 0.82 & 0.81 & 1 & 1 & 0.87 & 0.83 & 1 & 1 \\
\hline P6 & 0.87 & 0.91 & 1 & 0.87 & 1 & 1 & 1 & 1 & 1 & 0.84 & 0.90 & 0.76 & 1 & 1 & 1 & 0.95 & 1 & 1 & 1 & 1 & 1 & 1 \\
\hline P7 & 0.97 & 0.94 & 1 & 0.93 & 1 & 0.91 & 1 & 1 & 1 & 0.92 & 0.92 & 0.95 & 1 & 1 & 0.92 & 0.94 & 1 & 1 & 0.95 & 0.94 & 1 & 1 \\
\hline P8 & 0.55 & 0.69 & 1 & 0.52 & 1 & 0.56 & 1 & 1 & 1 & 0.48 & 0.57 & 0.50 & 1 & 1 & 0.67 & 0.64 & 1 & 1 & 0.74 & 0.67 & 1 & 1 \\
\hline P9 & 0.35 & 0.66 & 1 & 0.41 & 1 & 0.32 & 1 & 1 & 1 & 0.37 & 0.51 & 0.33 & 1 & 1 & 0.59 & 0.62 & 1 & 1 & 0.72 & 0.64 & 1 & 1 \\
\hline P10 & 0.68 & 0.85 & 0.68 & 0.99 & 0.68 & 0.85 & 0.68 & 0.68 & 0.68 & 1 & 0.95 & 0.80 & 0.68 & 0.68 & 0.83 & 0.91 & 0.68 & 0.68 & 0.87 & 0.90 & 0.68 & 0.68 \\
\hline P11 & 0.73 & 0.85 & 0.78 & 0.98 & 0.78 & 0.88 & 0.78 & 0.78 & 0.78 & 0.98 & 1 & 0.76 & 0.78 & 0.78 & 0.96 & 0.99 & 0.78 & 0.78 & 0.98 & 0.99 & 0.78 & 0.78 \\
\hline P12 & 0.79 & 0.70 & 0.63 & 0.86 & 0.63 & 0.50 & 0.63 & 0.63 & 0.63 & 0.89 & 0.85 & 1 & 0.63 & 0.63 & 0.63 & 0.84 & 0.63 & 0.63 & 0.80 & 0.82 & 0.63 & 0.63 \\
\hline P13 & 0.39 & 0.54 & 1 & 0.39 & 1 & 0.26 & 1 & 1 & 1 & 0.36 & 0.54 & 0.31 & 1 & 1 & 0.63 & 0.67 & 1 & 1 & 0.76 & 0.69 & 1 & 1 \\
\hline P14 & 0.85 & 0.89 & 1 & 0.84 & 1 & 0.81 & 1 & 1 & 1 & 0.83 & 0.86 & 0.83 & 1 & 1 & 0.88 & 0.89 & 1 & 1 & 0.92 & 0.90 & 1 & 1 \\
\hline P15 & 0.77 & 0.77 & 0.79 & 0.92 & 0.79 & 0.92 & 0.79 & 0.79 & 0.79 & 0.92 & 0.96 & 0.72 & 0.79 & 0.79 & 1 & 0.97 & 0.79 & 0.79 & 0.98 & 0.98 & 0.79 & 0.79 \\
\hline P16 & 0.24 & 0.55 & 0.69 & 0.87 & 0.69 & 0.53 & 0.69 & 0.69 & 0.69 & 0.87 & 0.96 & 0.37 & 0.69 & 0.69 & 0.87 & 1 & 0.69 & 0.69 & 0.96 & 0.99 & 0.69 & 0.69 \\
\hline P17 & 0.35 & 0.56 & 1 & 0.40 & 1 & 0.26 & 1 & 1 & 1 & 0.36 & 0.54 & 0.28 & 1 & 1 & 0.63 & 0.67 & 1 & 1 & 0.76 & 0.69 & 1 & 1 \\
\hline P18 & 0.23 & 0.52 & 1 & 0.36 & 1 & 0.23 & 1 & 1 & 1 & 0.33 & 0.49 & 0.26 & 1 & 1 & 0.57 & 0.62 & 1 & 1 & 0.69 & 0.63 & 1 & 1 \\
\hline P19 & 0.78 & 0.82 & 0.90 & 0.90 & 0.90 & 0.85 & 0.90 & 0.90 & 0.90 & 0.90 & 0.96 & 0.77 & 0.90 & 0.90 & 0.98 & 0.99 & 0.90 & 0.90 & 1 & 1 & 0.90 & 0.90 \\
\hline P20 & 0.76 & 0.85 & 0.86 & 0.96 & 0.86 & 0.86 & 0.86 & 0.86 & 0.86 & 0.96 & 0.99 & 0.78 & 0.86 & 0.86 & 0.97 & 0.99 & 0.86 & 0.86 & 1 & 1 & 0.86 & 0.86 \\
\hline P21 & 0.45 & 0.66 & 1 & 0.53 & 1 & 0.44 & 1 & 1 & 1 & 0.51 & 0.63 & 0.46 & 1 & 1 & 0.69 & 0.72 & 1 & 1 & 0.78 & 0.73 & 1 & 1 \\
\hline P22 & 0.51 & 0.63 & 1 & 0.69 & 1 & 0.60 & 1 & 1 & 1 & 0.68 & 0.79 & 0.52 & 1 & 1 & 0.84 & 0.87 & 1 & 1 & 0.89 & 0.88 & 1 & 1 \\
\hline
\end{tabular}

conditions that maximize the extraction of a certain compound (compounds $1,3,0,7,8,9,12,13,14,17,18,21$ and 22) do not favour at all the extraction of the other one (compounds 2 and 4 ).

Using the complete dataset of Table 4 and performing a multi-objective optimization problem using an appropriate clustering algorithm, different clusters of phenolic compounds whose maximum response values are obtained under similar HHP extraction conditions were created. The results of HCA are presented in Fig. 5. In the HCA dendrogram, the shorter distance between compounds indicates a higher similarity in terms of conditions that favour their extraction and the compounds clustered into the same group are better extracted under similar HHP conditions. Three significant clusters (C1, C2 and C3) were generated. C1 and C3 were also be divided in two ( $\mathrm{a}$ and $\mathrm{b}$ ) pertinent subgroups. Additionally, other less relevant subgroups were created in $\mathrm{C} 2, \mathrm{C} 1 \mathrm{~b}$ and $\mathrm{C} 3 \mathrm{~b}$, but they can be considered as a residual noise produced by the algorithm.

- Cluster C1 included the compounds 15, 16, 11 and 10. Meanwhile, compound 15 was subdivided in C1a and compounds 16, 11 and 10

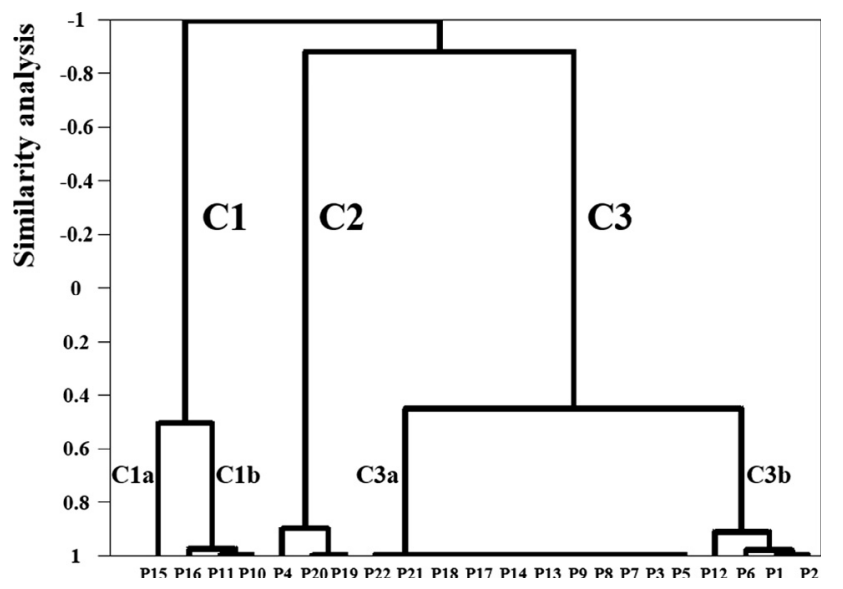

Fig. 5. Results of the hierarchical cluster analysis of phenolic compounds according to the HHP conditions that maximize their extraction from watercress. were grouped in $\mathrm{C} 1 \mathrm{~b}$. The extraction of these compounds was maximized by medium $t$, high $P$ and medium $S$ (Table 3 and Fig. 3). The subgroups $\mathrm{C} 1 \mathrm{a}$ and $\mathrm{C} 1 \mathrm{~b}$ were mainly differentiated by the $t$ values.

- Cluster C2 included the compounds 4, 20 and 19. No significant subgroups were created. The extraction of these compounds was favoured by medium $t$, high $P$ and medium-large $S$ values.

- Cluster C3 included the compounds 22, 21, 18, 17, 14, 13, 9, 8, 7, 3, $5,12,6,1$ and 2 , which were subdivided in C3a e C3b. The extraction of the compounds in C3a was maximized when using low $t$, high $P$ and high $S$. On the other hand, the compounds in C3b exhibited a broad set of conditions with no clear interconnections between each other.

Although it was expected that compounds with similar chemical characteristics would exhibit comparable optimal extraction conditions, no clear similarity was detected between the created groups of compounds and the conditions that maximize their extraction. However, this HCA analysis was an interesting and innovative approach in the field of extraction of high added-value compound from natural sources (something not seen in this type of studies), since it allowed grouping the phenolic compounds into different clusters according to the HHP conditions that favour their recovery from watercress, which can be very useful from a practical point of view.

\section{Conclusions}

As far as we know, this is the first study regarding the optimization of the extraction of phenolic compounds from watercress by HHP using RSM, a suitable statistical tool that allowed reduce the number of experimental trials and evaluate interactions among variables. The suitability of this cold extraction method (combining the independent variables $t, P$ and $S$ in a five-level CCCD design) was demonstrated. The developed polynomial response models were statistically validated and expressed as 2D and 3D surface plots to better visualize the effects on extraction yield and individual and grouped phenolic compounds (a total of 29 response variables). A good agreement between 
experimental and theoretical results was observed. In general, the recovery of phenolic compounds was maximized when high pressures, high ethanol concentrations and short extraction times were applied, which validate this cold extraction method as a very promising technique compared to the time-consuming conventional methods. This study also highlighted watercress as being an interesting source of phytochemicals, namely phenolic acids and flavonoids.

\section{Acknowledgements}

The authors are grateful to the Foundation for Science and Technology (FCT) of Portugal and FEDER under Programme PT2020 for financial support to CIMO (UID/AGR/00690/2013); to FCT/MEC for financial support to REQUIMTE/LAQV (UID/QUI/50006/2013 - POCI/ 01/0145/FERDER/007265) through national funds and co-financed by FEDER under Programme PT2020; to FCT/MEC for the financial support to the QOPNA research Unit (FCT/ID/QUI/00062/2013), through national funds and where applicable co-financed by the FEDER, within the PT2020 Partnership Agreement; to FCT for the grant attributed to J. Pinela (SFRH/BD/92994/2013) through the Programa Operacional Capital Humano (POCH) supported by the European Social Fund and National Funds of MCTES (Ministério da Ciência, Tecnologia, e Ensino Superior); to FCT for L. Barros research contract; and to the Xunta de Galicia for financial support to M.A. Prieto. The authors are also grateful to the Interreg España-Portugal for financial support through the project 0377_Iberphenol_6_E.

\section{References}

[1] C. Caleja, A. Ribeiro, L. Barros, J.C.M. Barreira, A.L. Antonio, M. Beatriz, P.P. Oliveira, M.F. Barreiro, I.C.F.R. Ferreira, Cottage cheeses functionalized with fennel and chamomile extracts: Comparative performance between free and microencapsulated forms, Food Chem. 199 (2016) 720-726.

[2] C. Pereira, L. Barros, M.J. Alves, L. Pereira, C. Santos-Buelga, I.C.F.R. Ferreira, Phenolic profile and antimicrobial activity of different dietary supplements based on Cochlospermum angolensis Welw, Ind. Crops Prod. 74 (2015) 412-416.

[3] O. Taofiq, S. Heleno, R. Calhelha, M. Alves, L. Barros, M. Barreiro, A.M. González Paramás, I.C.F.R. Ferreira, Development of mushroom-based cosmeceutical formulations with anti-inflammatory, anti-tyrosinase, antioxidant, and antibacterial properties in food and pharmaceutical industries has increased year by year, Molecules 21 (2016) 1372.

[4] I.C.F.R. Ferreira, N. Martins, L. Barros, Phenolic compounds and its bioavailability: In vitro bioactive compounds or health promoters? in: F. Toldrá (Ed.), Adv. Food Nutr. Res. Academic Press, 2017, pp. 1-44.

[5] J. Pinela, M.A. Prieto, A.M. Carvalho, M.F. Barreiro, M.B.P.P. Oliveira, L. Barros, I.C.F.R. Ferreira, Microwave-assisted extraction of phenolic acids and flavonoids and production of antioxidant ingredients from tomato: A nutraceutical-oriented optimization study, Sep. Purif. Technol. 164 (2016) 114-124.

[6] C. Caleja, L. Barros, M.A. Prieto, M.F. Barreiro, M.B.P.P. Oliveira, I.C.F.R. Ferreira, Extraction of rosmarinic acid from Melissa officinalis L. by heat-, microwave- and ultrasound-assisted extraction techniques: A comparative study through response surface analysis, Sep. Purif. Technol. 186 (2017) 297-308.

[7] V. Vieira, M.A. Prieto, L. Barros, J.A.P. Coutinho, O. Ferreira, I.C.F.R. Ferreira, Optimization and comparison of maceration and microwave extraction systems for the production of phenolic compounds from Juglans regia L. for the valorization of walnut leaves, Ind. Crops Prod. 107 (2017) 341-352.

[8] J.V. Higdon, B. Delage, D.E. Williams, R.H. Dashwood, Cruciferous vegetables and human cancer risk: epidemiologic evidence and mechanistic basis, Pharmacol. Res. 55 (2007) 224-236

[9] P. Ravasco, D.R. João, I. Rowland, N. Giallourou, J. Swann, L.M. Dias, J. Sain, S. Rothwell, M. Fogarty, N. Harbourne, MON-LB019: Does watercress influence phase angle in breast cancer patients during radiotherapy? Clin. Nutr. 34 (2015) S257.

[10] P. Ravasco, D. João, M. Jorge, I. Rowland, N. Giallourou, J. Swann, L.M. Dias, J. Sain, S. Rothwell, M. Fogarty, N. Harbourne, MON-LB027: Watercress in breast cancer patients undergoing radiotherapy: relevance in DNA damage of dermatitis? Clin. Nutr. 34 (2015) S260-S261.

[11] C.I.R. Gill, S. Haldar, L.A. Boyd, R. Bennett, J. Whiteford, M. Butler, J.R. Pearson, I. Bradbury, I.R. Rowland, Watercress supplementation in diet reduces lymphocyte DNA damage and alters blood antioxidant status in healthy adults, Am. J. Clin. Nutr. 85 (2007) 504-510.

[12] V. Spínola, J. Pinto, P.C. Castilho, In vitro studies on the effect of watercress juice on digestive enzymes relevant to type 2 diabetes and obesity and antioxidant activity, J. Food Biochem. 41 (2016) e12335.

[13] A. Zeb, Phenolic profile and antioxidant potential of wild watercress (Nasturtium officinale), Springerplus. 4 (2015) 714.

[14] J. Pinela, L. Barros, J.C.M. Barreira, A.M. Carvalho, M.B.P.P. Oliveira, C. Santos-
Buelga, I.C.F.R. Ferreira, Postharvest changes in the phenolic profile of watercress induced by post-packaging irradiation and modified atmosphere packaging, Unpublished results.

[15] L.A. Boyd, M.J. McCann, Y. Hashim, R.N. Bennett, C.I.R. Gill, I.R. Rowland, Assessment of the anti-genotoxic, anti-proliferative, and anti-metastatic potential of crude watercress extract in human colon cancer cells., Nutr. Cancer. 55 (2006) 232-241.

[16] P. Rose, K. Faulkner, G. Williamson, R. Mithen, 7-Methylsulfinylheptyl and 8-methylsulfinyloctyl isothiocyanates from watercress are potent inducers of phase II enzymes, Carcinogenesis 21 (2000) 1983-1988.

[17] P. Rose, Q. Huang, C.N. Ong, M. Whiteman, Broccoli and watercress suppress matrix metalloproteinase-9 activity and invasiveness of human MDA-MB-231 breast cancer cells, Toxicol. Appl. Pharmacol. 209 (2005) 105-113.

[18] H. Mújica-Paz, A. Valdez-Fragoso, C.T. Samson, J. Welti-Chanes, J.A. Torres, Highpressure processing technologies for the pasteurization and sterilization of foods, Food Bioprocess Technol. 4 (2011) 969-985.

[19] M. Hernández-Carrión, I. Hernando, A. Quiles, High hydrostatic pressure treatment as an alternative to pasteurization to maintain bioactive compound content and texture in red sweet pepper, Innov. Food Sci. Emerg. Technol. 26 (2014) 76-85.

[20] J. Pinela, I.C.F.R. Ferreira, Nonthermal physical technologies to decontaminate and extend the shelf-life of fruits and vegetables: Trends aiming at quality and safety, Crit. Rev. Food Sci. Nutr. 57 (2017) 2095-2111.

[21] S.M. Castro, J.A. Saraiva, High-pressure processing of fruits and fruit products, in: D.-W. Sun (Ed.), Emerg. Technol. Food Process., second ed., Academic Press, 2014 pp. 65-76.

[22] I.F. Strati, E. Gogou, V. Oreopoulou, Enzyme and high pressure assisted extraction of carotenoids from tomato waste, Food Bioprod. Process. 94 (2015) 668-674.

[23] L. Wang, Energy efficiency technologies for sustainable food processing, Energy Effic. 7 (2014) 791-810.

[24] H.-W. Huang, C.-P. Hsu, B.B. Yang, C.-Y. Wang, Advances in the extraction of natural ingredients by high pressure extraction technology, Trends Food Sci. Technol. 33 (2013) 54-62.

[25] X. Jun, Z. Shuo, L. Bingbing, Z. Rui, L. Ye, S. Deji, Z. Guofeng, Separation of major catechins from green tea by ultrahigh pressure extraction, Int. J. Pharm. 386 (2010) 229-231.

[26] K.N. Prasad, E. Yang, C. Yi, M. Zhao, Y. Jiang, Effects of high pressure extraction on the extraction yield, total phenolic content and antioxidant activity of longan fruit pericarp, Innov. Food Sci. Emerg. Technol. 10 (2009) 155-159.

[27] W.M. Elamin, J.B. Endan, Y.A. Yosuf, R. Shamsudin, A. Ahmedov, High pressure processing technology and equipment evolution: A review, J. Eng. Sci. Technol. Rev. 8 (2015) 75-83.

[28] V. Briones-Labarca, M. Plaza-Morales, C. Giovagnoli-Vicuña, F. Jamett, High hydrostatic pressure and ultrasound extractions of antioxidant compounds, sulforaphane and fatty acids from Chilean papaya (Vasconcellea pubescens) seeds: Effects of extraction conditions and methods, LWT - Food Sci. Technol. 60 (2015) 525-534.

[29] Z. Shouqin, X. Jun, W. Changzheng, High hydrostatic pressure extraction of flavonoids from propolis, J. Chem. Technol. Biotechnol. 80 (2005) 50-54.

[30] E.M.C. Alexandre, P. Araújo, M.F. Duarte, V. de Freitas, M. Pintado, J.A. Saraiva, High-pressure assisted extraction of bioactive compounds from industrial fermented fig by-product, J. Food Sci. Technol. 54 (2017) 2519-2531.

[31] R.N. Pereira, A.A. Vicente, Environmental impact of novel thermal and non-thermal technologies in food processing, Food Res. Int. 43 (2010) 1936-1943.

[32] E.M.C. Alexandre, P. Araújo, M.F. Duarte, V. de Freitas, M. Pintado, J.A. Saraiva, Experimental design, modeling, and optimization of high-pressure-assisted extraction of bioactive compounds from pomegranate peel, Food Bioprocess Technol. 10 (2017) 886-900.

[33] R. Casquete, S.M. Castro, M.C. Villalobos, M.J. Serradilla, R.P. Queirós, J.A. Saraiva, M.G. Córdoba, P. Teixeira, High pressure extraction of phenolic compounds from citrus peels, High Press. Res. 34 (2014) 447-451.

[34] M. Corrales, A.F. García, P. Butz, B. Tauscher, Extraction of anthocyanins from grape skins assisted by high hydrostatic pressure, J. Food Eng. 90 (2009) 415-421.

[35] X. Jun, Caffeine extraction from green tea leaves assisted by high pressure processing, J. Food Eng. 94 (2009) 105-109.

[36] H.-S. Lee, H.J. Lee, H.J. Yu, D.W. Ju, Y. Kim, C.-T. Kim, C.-J. Kim, Y.-J. Cho, N. Kim, S.-Y. Choi, H.J. Suh, A comparison between high hydrostatic pressure extraction and heat extraction of ginsenosides from ginseng (Panax ginseng CA Meyer), J. Sci. Food Agric. 91 (2011) 1466-1473.

[37] J. Xi, S. Luo, The mechanism for enhancing extraction of ferulic acid from Radix Angelica sinensis by high hydrostatic pressure, Sep. Purif. Technol. 165 (2016) 208-213.

[38] S.M.F. Bessada, J.C.M. Barreira, L. Barros, I.C.F.R. Ferreira, M.B.P.P. Oliveira, Phenolic profile and antioxidant activity of Coleostephus myconis (L.) Rchb.f.: An underexploited and highly disseminated species, Ind. Crops Prod. 89 (2016) 45-51.

[39] J. Santos, M.B.P.P. Oliveira, E. Ibáñez, M. Herrero, Phenolic profile evolution of different ready-to-eat baby-leaf vegetables during storage, J. Chromatogr. A. 1327 (2014) 118-131.

[40] A. Martínez-Sánchez, A. Gil-Izquierdo, M.I. Gil, F. Ferreres, A comparative study of flavonoid compounds, vitamin C, and antioxidant properties of baby leaf Brassicaceae species, J. Agric. Food Chem. 56 (2008) 2330-2340.

[41] M. Ranic, M. Nikolic, M. Pavlovic, A. Buntic, S. Siler-Marinkovic, S. DimitrijevicBrankovic, Optimization of microwave-assisted extraction of natural antioxidants from spent espresso coffee grounds by response surface methodology, J. Clean. Prod. 80 (2014) 69-79.

[42] S. Zhang, H. Bi, C. Liu, Extraction of bio-active components from Rhodiola sachalinensis under ultrahigh hydrostatic pressure, Sep. Purif. Technol. 57 (2007) 277-282. 